\title{
Spacetime and universal soft modes: Black holes and beyond
}

\author{
Yasunori Nomura ${ }^{\circ}$ \\ Berkeley Center for Theoretical Physics, Department of Physics, University of California, \\ Berkeley, California 94720, USA; \\ Theoretical Physics Group, Lawrence Berkeley National Laboratory, Berkeley, California 94720, USA; \\ and Kavli Institute for the Physics and Mathematics of the Universe (WPI), UTIAS, \\ The University of Tokyo, Kashiwa, Chiba 277-8583, Japan
}

(Received 25 August 2019; accepted 27 February 2020; published 25 March 2020)

\begin{abstract}
Recently, a coherent picture of the quantum mechanics of an evaporating black hole has been presented which reconciles unitarity with the predictions of the equivalence principle. The thermal nature of a black hole as viewed in a distant reference frame arises from entanglement between the hard and soft modes generated by the chaotic dynamics at the string scale. In this paper, we elaborate on this picture, particularly emphasizing the importance of the chaotic nature of the string (UV) dynamics across all low-energy species in generating large (IR) spacetime behind the horizon. Implications of this UV/IR relation include $O(1)$ breaking of global symmetries at the string scale and a self-repair mechanism of black holes restoring the smoothness of their horizons. We also generalize the framework to other systems, including Rindler, de Sitter, and asymptotically flat spacetimes, and find a consistent picture in each case. Finally, we discuss the origin of the particular construction adopted in describing the black hole interior as well as the outside of a de Sitter horizon. We argue that the construction is selected by the quantum-to-classical transition, in particular, the applicability of the Born rule in a quantum mechanical world.
\end{abstract}

DOI: 10.1103/PhysRevD.101.066024

\section{INTRODUCTION}

Ever since the thermodynamics of a black hole was discovered [1,2], it has been a key element in advancing our understanding of quantum gravity. On one hand, the fact that the entropy of a black hole is proportional to its horizon area has led to the idea of holography [3-5], which is elegantly realized in the AdS/CFT correspondence [6]. On the other hand, the fact that a black hole has a nonzero temperature has led to confusion about the consistency between quantum mechanics and general relativity [7-9]. It is widely believed that a solution to this problem would give us major insight into how quantum gravity works at the fundamental level.

In recent work, we have presented a coherent picture of the quantum mechanics of an evaporating black hole [10], building on the tools and ideas developed earlier [11-13] (for more complete references, see Ref. [10]). From the point of view of a distant observer, the thermality of a black hole arises because a vast majority of degrees of freedom - which we call soft modes-become temporarily unobservable

Published by the American Physical Society under the terms of the Creative Commons Attribution 4.0 International license. Further distribution of this work must maintain attribution to the author(s) and the published article's title, journal citation, and DOI. Funded by SCOAP ${ }^{3}$. because of the large redshift. In an ordinary statistical mechanical system, an equilibrated state is described by a thermal density matrix if we focus only on a small subset, even if the entire state is pure. The same occurs for a black hole. Since the degrees of freedom described by semiclassical theory-which are the complement of the soft modes and called hard modes - are only a small subset of the whole, their state is given by a thermal density matrix if the black hole is in its ground state. Unlike many other applications in statistical mechanics, however, a useful separation between the relevant subsystem and the rest in the black hole case is given in momentum space, rather than in position space. The thermality of the hard modes then arises from the entanglement in momentum space, i.e., between the hard and soft modes, resulting from the energy constraint.

The purpose of this paper is threefold. First, we elucidate the picture of Ref. [10], particularly emphasizing the role played by the chaotic dynamics at the string scale. We find an intriguing relation between the chaotic dynamics in the ultraviolet (UV) and the emergence of smooth spacetime in the infrared (IR). This relation suggests that the string scale dynamics is chaotic across all low-energy species and breaks all (linearly realized) global symmetries with $O(1)$ strength at the string scale. We also discuss why the present framework does not suffer from the "Born rule problem" of Ref. [14] and describe a mechanism with which a black hole "self-repairs" itself to restore the smooth horizon. 
We then generalize the framework to other spacetimes, including Rindler, de Sitter, and asymptotically flat spacetimes. We obtain a consistent picture in each case. In particular, in de Sitter spacetime, a construction similar to the black hole interior allows for describing the situation in which semiclassical information going outside the de Sitter horizon is retrieved later when the system tunnels out of the original de Sitter vacuum. Soft modes play an important role in this description. In the flat space limit, these modes decouple from the dynamics occurring in a finite spacetime region. They, however, seem to be related to the existence of an infinitedimensional asymptotic symmetry group, including the (diagonal) Bondi-Metzner-Sachs (BMS) group [15-17].

Finally, we discuss the origin of the particular construction adopted in describing the black hole interior and the outside of a de Sitter horizon. We argue that this construction is selected by the quantum-to-classical transition, specifically by the requirement of making most manifest the observables to which the Born rule can be applied. While this issue is irrelevant for asymptotically flat or $\mathrm{AdS}$ spacetime, it can become very important when describing a system that is (effectively) finite dimensional, such as the black hole interior and cosmological spacetimes.

The organization of this paper is as follows. In Sec. II, we describe the framework of Ref. [10], emphasizing its salient feature of the UV/IR relation. This section forms the basis for the rest of the paper. In Sec. III, we generalize the framework to other spacetimes, including Rindler, de Sitter, and asymptotically flat spacetimes. In Sec. IV, we discuss the issue of observables in a quantum theory, where the emergence of the construction relevant for the black hole interior is elucidated from the viewpoint of the applicability of the Born rule.

The framework discussed in this paper is consistent with and, in fact, complementary to recent analyses of an evaporating black hole in the AdS/CFT correspondence $[18,19] .{ }^{1}$ Our analysis elucidates the very appearance of the interior region-in particular, how the effective second exterior degrees of freedom emerge-from a microscopic point of view. It illuminates what each element in the AdS/CFT-based models, involving a large AdS black hole coupled to an external auxiliary system, corresponds to in a more realistic black hole. The general analysis presented here also sheds light on aspects of spacetime in quantum gravity in broader contexts.

Throughout the paper, $l_{\mathrm{P}}$ denotes the Planck length. We adopt natural units $c=\hbar=1$.

\section{BLACK HOLE INTERIOR AND UNIVERSAL SOFT MODES}

In this section, we describe the framework of Ref. [10], elucidating several points that play central roles in our

\footnotetext{
${ }^{1}$ For important differences between the case of a flat space black hole discussed in this paper and the case of Refs. [18,19] corresponding to a large AdS black hole, see Ref. [20].
}

discussion. We particularly emphasize the importance of the chaotic nature of string dynamics across all low-energy species and the intriguing relation between the dynamics in the UV (string scale) and the emergence of smooth spacetime in the IR. We also explain why the Born rule problem of Ref. [14] does not apply to the current framework.

We will focus on Schwarzschild black holes in fourdimensional asymptotically flat spacetime (or small black holes in four-dimensional asymptotically AdS spacetime), though the restriction on specific spacetime dimensions or on nonrotating, noncharged black holes is not essential.

\section{A. Evaporating black hole and its interior}

We begin our discussion with the description of a black hole as viewed from a distant reference frame. This corresponds to a semiclassical description that uses a metric covering the exterior of the black hole, i.e., the one using the Schwarzschild time (or time analogous to it). This description arises naturally in holography-it is the description obtained from the boundary picture through simple bulk reconstruction of renormalization-group/ tensor-network type [21] and, as such, is unitary. On the other hand, the description suitable for an infalling observer corresponding to the Kruskal extension is obtained only effectively, as we will see later.

A key observation of Ref. [10] is that the thermal nature of a black hole in a distant description arises from entanglement between hard and soft modes of low-energy quantum fields. $^{2}$ A mode of a low-energy quantum field in the zone region (also called the thermal atmosphere)

$$
r_{\mathrm{s}} \leq r \leq r_{\mathrm{z}}
$$

is classified as a hard or soft mode, depending on whether its frequency $\omega$, as measured in the asymptotic region, is larger or smaller than

$$
\Delta \approx O\left(\frac{1}{M l_{\mathrm{P}}^{2}}\right) .
$$

Here, $r_{\mathrm{z}} \approx 3 M l_{\mathrm{P}}^{2}$, and $r_{\mathrm{s}}$ is the location of the stretched horizon [22] given by $^{3}$

$$
r_{\mathrm{s}}-2 M l_{\mathrm{P}}^{2} \sim \frac{l_{\mathrm{s}}^{2}}{M l_{\mathrm{P}}^{2}} .
$$

In a distant description, the classical spacetime picture is applicable only outside the stretched horizon, and its location is determined by the condition that the proper distance from the mathematical horizon $r=2 M l_{\mathrm{P}}^{2}$ is of order the string length, $l_{\mathrm{s}}$.

\footnotetext{
${ }^{2}$ Here and below, low-energy fields mean quantum fields existing below the string scale, $1 / l_{\mathrm{s}}$.

${ }^{3}$ In this paper, we use the $\sim$ symbol to mean equality up to numerical coefficients.
} 
The quantity $\Delta$ in Eq. (2) is taken to be somewhat, e.g., by a factor of $O(10)$, larger than the Hawking temperature

$$
T_{\mathrm{H}}=\frac{1}{8 \pi M l_{\mathrm{P}}^{2}} .
$$

The separation of the modes described above is motivated by the fact that the configuration of soft modes cannot be determined operationally by a physical probe within the characteristic timescale with which the internal state of the system varies. Note that $\Delta$ is the inverse of the timescale for single Hawking emission, so that the uncertainty principle prevents us from specifying the energy of the black hole state better than that. Below, we will assume that the energy (mass) of a black hole is specified with this maximal precision.

A state of a black hole-representing the state of the system in the black hole zone region-is given in its semiclassical vacuum by a generic state consistent with the energy constraint imposed on the region. In particular, in an idealized limit in which the black hole is isolated from the environment, a state of a black hole of mass $M$ is given by

$$
|\Psi(M)\rangle=\sum_{n} \sum_{i_{n}=1}^{\mathcal{N}\left(M-E_{n}\right)} c_{n i_{n}}\left|\left\{n_{\alpha}\right\}\right\rangle\left|\psi_{i_{n}}\left(M-E_{n}\right)\right\rangle,
$$

where $\left|\left\{n_{\alpha}\right\}\right\rangle$ are orthonormal states of the hard modes with $n \equiv\left\{n_{\alpha}\right\}$ representing the set of all occupation numbers; $\alpha$ collectively denotes the species, frequency, and angularmomentum quantum numbers of a mode, and $E_{n}$ is the energy of the state $\left|\left\{n_{\alpha}\right\}\right\rangle$ as measured in the asymptotic region (with precision $\Delta) .\left|\psi_{i_{n}}\left(M-E_{n}\right)\right\rangle$ are orthonormal states of the soft modes which have energy $M-E_{n}$, with the index $i_{n}$ running from 1 to $\mathcal{N}\left(M-E_{n}\right)$, where

$$
\mathcal{N}(M)=e^{S_{\mathrm{BH}}(M)} \frac{\Delta}{M}=e^{4 \pi M^{2} l_{\mathrm{P}}^{2}} \frac{\Delta}{M} .
$$

Below, we ignore the logarithmic correction of order $\ln (\Delta / M)$ to the entropy, identifying $\mathcal{N}(M)$ as the density of states $e^{S_{\mathrm{BH}}(M)}$. We also assume that all the states are normalized; for example, we assume $\sum_{n} \sum_{i_{n}=1}^{\mathcal{N}\left(M-E_{n}\right)}\left|c_{n i_{n}}\right|^{2}=1$ in Eq. (5). Note that the total entropy of the state of the form in Eq. (5) is

$$
\ln \left[\sum_{n} e^{S_{\mathrm{BH}}\left(M-E_{n}\right)}\right] \approx S_{\mathrm{BH}}(M),
$$

so that the logarithm of the number of independent black hole microstates is given by the standard Bekenstein-Hawking entropy.

In a realistic setup, a black hole state is entangled with the environment which generally involves Hawking radiation emitted earlier. The state of the total system is then given by

$$
|\Psi(M)\rangle=\sum_{n} \sum_{i_{n}=1}^{\mathcal{N}\left(M-E_{n}\right)} \sum_{a} c_{n i_{n} a}\left|\left\{n_{\alpha}\right\}\right\rangle\left|\psi_{i_{n}}\left(M-E_{n}\right)\right\rangle\left|\phi_{a}\right\rangle,
$$

where $\left|\phi_{a}\right\rangle$ represents the state of the system in the far region $r>r_{\mathrm{z}}$. Note that states of this form appear in a distant description of the system, which corresponds to the boundary description in a holographic theory, so that their evolution is unitary. The thermal nature of a black hole arises because, within the zone, semiclassical theory describes microscopic dynamics of only the hard modes. Indeed, tracing out soft modes in Eq. (8) yields

$$
\begin{aligned}
& \operatorname{Tr}_{\text {soft }}|\Psi(M)\rangle\langle\Psi(M)| \\
& \quad=\frac{1}{\sum_{n} e^{-\frac{E_{n}}{T_{\mathrm{H}}}} \sum_{n} e^{-\frac{E_{n}}{T_{\mathrm{H}}}}\left|\left\{n_{\alpha}\right\}\right\rangle\left\langle\left\{n_{\alpha}\right\}\right| \otimes \rho_{\phi, n},}
\end{aligned}
$$

where we have assumed that the coefficients $c_{n i_{n} a}$ take generic values in the spaces of the hard and soft modes; i.e., black hole states are generic. $\rho_{\phi, n}$ are (n-dependent) reduced density matrices for the far modes.

A small object in the zone region, with the characteristic size $d$ in the angular directions much smaller than the horizon $d \ll M l_{\mathrm{P}}^{2}$, can be described by annihilation and creation operators acting only on the hard modes [10]

$$
\begin{aligned}
& b_{\gamma}=\sum_{n} \sqrt{n_{\gamma}}\left|\left\{n_{\alpha}-\delta_{\alpha \gamma}\right\}\right\rangle\left\langle\left\{n_{\alpha}\right\}\right|, \\
& b_{\gamma}^{\dagger}=\sum_{n} \sqrt{n_{\gamma}+1}\left|\left\{n_{\alpha}+\delta_{\alpha \gamma}\right\}\right\rangle\left\langle\left\{n_{\alpha}\right\}\right| .
\end{aligned}
$$

What is the fate of such an object falling toward the black hole?

In a distant/boundary description, in which the evolution of a state can be unitary for arbitrarily long time, a small object falling into the black hole first becomes excitations of the stretched horizon, whose information will then be dissipated into the state of the soft modes and eventually sent back to ambient space by Hawking emission. This description, however, is not suitable for finding what the object falling into the black hole will actually see. Because of a large relative boost between the object and the distant frame, which formally becomes infinite as the object approaches the horizon, macroscopic time experienced by the object is mapped to an extremely short time when measured by a stationary observer at the location of the object. This implies that the experience of the object occurs "instantaneously" in a distant description (of order the cutoff time for an observer at $r=r_{\mathrm{s}}$ ). Understanding it, therefore, requires time evolution different from the boundary one, specifically an evolution associated with the proper time of the object. 
Such a picture-an infalling description-is obtained after coarse-graining the soft (and associated far) mode degrees of freedom [10-12], which cannot be discriminated by a fallen object in a timescale available to it. Suppose that at a boundary time $t_{*}$, the state of the system is given by Eq. (8) with $c_{n i_{n} a}$ taking generic values in the $n$ and $i_{n}$ spaces. ${ }^{4}$ We can then erect an effective theory based on this state by coarse-graining the soft and far modes

$$
\left.\left.\sum_{i_{n}=1}^{\mathcal{N}\left(M-E_{n}\right)} \sum_{a} c_{n i_{n} a}\left|\psi_{i_{n}}\left(M-E_{n}\right)\right\rangle\left|\phi_{a}\right\rangle \rightarrow \frac{e^{-\frac{E_{n}}{2 T_{\mathrm{H}}}}}{\sqrt{\sum_{n} e^{-\frac{E_{n}}{T_{\mathrm{H}}}}}} \|\left\{n_{\alpha}\right\}\right\rangle\right\rangle,
$$

where we have used the same label as the corresponding hard-mode state to specify the coarse-grained state, which we denote by the double ket symbol, and the coefficient in the right-hand side arises from the normalization condition for $\left.\left.\|\left\{n_{\alpha}\right\}\right\rangle\right\rangle$. The state in Eq. (8) in this effective theory is then given by

$$
\left.\left.\| \Psi(M)\rangle\rangle=\frac{1}{\sqrt{\sum_{n} e^{-\frac{E_{n}}{T_{H}}}}} \sum_{n} e^{-\frac{E_{n}}{2 T_{H}}}\left|\left\{n_{\alpha}\right\}\right\rangle \|\left\{n_{\alpha}\right\}\right\rangle\right\rangle,
$$

which takes the form of the standard thermofield double state in the two-sided black hole picture [25,26], although $\left|\left\{n_{\alpha}\right\}\right\rangle$ here represent the states only of the hard modes.

We emphasize that in order to obtain the correct Boltzmann-weight coefficients in Eqs. (12) and (13) $\propto e^{-E_{n} / 2 T_{\mathrm{H}}}$, it is important that the black hole has soft modes with the density of states given by $e^{S_{\mathrm{BH}}\left(E_{\text {soft }}\right)}$, and that the hard and soft modes are well scrambled, giving $c_{n i_{n} a}$ that take values statistically independent of $n .^{5}$ We also stress that the operation of coarse-graining, i.e., ignoring the detailed structure of $c_{n i_{n} a}$ 's, is different from tracing out degrees of freedom. It is this coarse-graining that leads to the apparent uniqueness of the infalling vacuum, despite the existence of exponentially many black hole microstates.

We can now define the "mirror operators" acting on the coarse-grained states

$$
\begin{aligned}
& \left.\left.\tilde{b}_{\gamma}=\sum_{n} \sqrt{n_{\gamma}} \|\left\{n_{\alpha}-\delta_{\alpha \gamma}\right\}\right\rangle\right\rangle\left\langle\left\{\left\{n_{\alpha}\right\} \|,\right.\right. \\
& \left.\tilde{b}_{\gamma}^{\dagger}=\sum_{n} \sqrt{n_{\gamma}+1} \|\left\{n_{\alpha}+\delta_{\alpha \gamma}\right\}\right\rangle\left\langle\left\langle\left\{n_{\alpha}\right\} \| .\right.\right.
\end{aligned}
$$

\footnotetext{
${ }^{4}$ In an asymptotically flat spacetime, the boundary time $t$ can be taken as the Schwarzschild time. In more general cases, $t$ can be a time parameter on the holographic screen [23,24].

${ }^{5}$ Note that even after the Page time [27], when the coarsegrained entropy of the emitted radiation is greater than the black hole entropy, the number of independent microstates that couple to the hard-mode state $\left|\left\{n_{\alpha}\right\}\right\rangle$ is still controlled by the density of soft-mode states, $e^{S_{\mathrm{BH}}\left(M-E_{n}\right)}$.
}

This implies that modes in the second exterior of the effective theory arise as (hard) quasiparticles generated by collective excitations of the soft modes as well as the far mode degrees of freedom entangling with them, including early Hawking radiation. Note that at the microscopic level, these operators act on both soft and far degrees of freedom. Indeed, tracing out the soft/far modes in Eq. (8), the remaining correlation between the hard and far/soft modes is essentially classical.

The mirror operators in Eqs. (14) and (15) allow us, together with the operators in Eqs. (10) and (11), to form the annihilation and creation operators for infalling modes

$$
\begin{aligned}
& a_{\xi}=\sum_{\gamma}\left(\alpha_{\xi \gamma} b_{\gamma}+\beta_{\xi \gamma} b_{\gamma}^{\dagger}+\zeta_{\xi \gamma} \tilde{b}_{\gamma}+\eta_{\xi \gamma} \tilde{b}_{\gamma}^{\dagger}\right), \\
& a_{\xi}^{\dagger}=\sum_{\gamma}\left(\beta_{\xi \gamma}^{*} b_{\gamma}+\alpha_{\xi \gamma}^{*} b_{\gamma}^{\dagger}+\eta_{\xi \gamma}^{*} \tilde{b}_{\gamma}+\zeta_{\xi \gamma}^{*} \tilde{b}_{\gamma}^{\dagger}\right),
\end{aligned}
$$

where $\xi$ is the label in which the frequency $\omega$ with respect to $t$ is traded with the frequency $\Omega$ associated with the infalling time, and $\alpha_{\xi \gamma}, \beta_{\xi \gamma}, \zeta_{\xi \gamma}$, and $\eta_{\xi \gamma}$ are the Bogoliubov coefficients calculable using the standard field theory method. The generator of time evolution in this description is then given by

$$
H=\sum_{\xi} \Omega a_{\xi}^{\dagger} a_{\xi}+H_{\mathrm{int}}\left(a_{\xi}, a_{\xi}^{\dagger}\right)
$$

This leads to the physics of a smooth horizon. The existence of these operators implies that there is a subsector in the original microscopic theory encoding the experience of an object after it crosses the horizon. (For further discussion, see Sec. IV.)

The effective theory erected as above is applicable only for a limited spacetime region [10]. Since the far modes are coarse-grained, the theory describes only physics within the causal domain of the union of the zone and its mirror regions on the $t=t_{*}$ hypersurface (the time at which the effective theory is erected). Furthermore, the fact that the soft modes are coarse-grained implies that the description is intrinsically semiclassical; i.e., it is valid only down to the length scale $l_{\mathrm{s}}$. This suggests that the singularity of a black hole may not be resolved; it may simply represent an intrinsic limitation coming from the fact that the theory of the interior is obtained by coarse-graining and hence describes a finite-dimensional, nonunitary system. The fact that an effective theory describes only a limited spacetime region also implies that the picture of the whole interior, as indicated by general relativity, can be obtained only by using multiple effective theories erected at different times (which are generally not independent). This is the sense in which the concept of the black hole interior emerges from the microscopic description of the black hole. 


\section{B. "Spacetime" and matter within low-energy fields}

A salient feature of the framework described above is that low-energy quantum fields contain both degrees of freedom associated with the Bekenstein-Hawking entropy (spacetime) and matter (excitations). One might be skeptical about this, but there are many arguments suggesting that it indeed gives a valid picture.

\section{The number of low-energy species}

Let us estimate the number of soft modes contained in a single low-energy field. This is done by integrating the entropy density

$$
s_{0}(r)=c T_{\mathrm{loc}}(r)^{3}
$$

over the zone region Eq. (1), where $c$ is a constant of $O(1)$, and

$$
T_{\mathrm{loc}}(r)=\frac{T_{\mathrm{H}}}{\sqrt{1-\frac{2 M l_{\mathrm{P}}^{2}}{r}}}
$$

is the local temperature measured at $r$. This gives

$$
S_{\mathrm{soft}, 0}=\int_{r_{\mathrm{s}}}^{r_{\mathrm{z}}} s_{0}(r) \frac{r^{2} d r d \Omega}{\sqrt{1-\frac{2 M l_{\mathrm{P}}^{2}}{r}}} \sim \frac{M^{2} l_{\mathrm{P}}^{4}}{l_{\mathrm{s}}^{2}} .
$$

Alternatively, one could directly count the number of modes excited. Specifically, a particle with angular momentum $L^{2}=\ell(\ell+1)$ costs the energy, as measured in the asymptotic region of

$$
\Delta \omega \sim \frac{\ell}{r},
$$

so that

$$
\frac{\Delta \omega}{T_{\mathrm{loc}}(r)} \sim \frac{\ell}{T_{\mathrm{H}} r} \sqrt{1-\frac{2 M l_{\mathrm{P}}^{2}}{r}} \sim \ell \sqrt{1-\frac{2 M l_{\mathrm{P}}^{2}}{r}} .
$$

Therefore, modes up to $\ell_{\max } \sim \sqrt{r /\left(r-2 M l_{\mathrm{P}}^{2}\right)}$ are effectively populated, giving the same result as Eq. (21),

$$
\left.S_{\mathrm{soft}, 0} \sim \sum_{\ell=0}^{\ell_{\max }}(2 \ell+1)\right|_{r=r_{\mathrm{s}}} \sim \frac{M l_{\mathrm{P}}^{2}}{r_{\mathrm{s}}-2 M l_{\mathrm{P}}^{2}} \sim \frac{M^{2} l_{\mathrm{P}}^{4}}{l_{\mathrm{s}}^{2}} .
$$

By going from the angular momentum to angular position bases, we find that most of the soft-mode degrees of freedom are located on the stretched horizon, with $O(1)$ degrees of freedom per string area $\sim l_{\mathrm{s}}^{2}$ for each low-energy field.

The total entropy of the soft modes is given by multiplying the number of low-energy fields $N$ to $S_{\text {soft }, 0}$,

$$
S_{\text {soft }} \sim N S_{\text {soft }, 0} \sim N \frac{M^{2} l_{\mathrm{P}}^{4}}{l_{\mathrm{s}}^{2}} .
$$

Using the relation expected in any theory of quantum gravity (see, e.g., Ref. [28])

$$
l_{\mathrm{P}}^{2} \sim \frac{l_{\mathrm{s}}^{2}}{N},
$$

we find that this indeed reproduces the BekensteinHawking entropy, up to an incalculable $O(1)$ factor

$$
S_{\mathrm{soft}} \sim M^{2} l_{\mathrm{P}}^{2} \sim S_{\mathrm{BH}} .
$$

This is consistent with the view that the BekensteinHawking entropy is mostly on the stretched horizon with the surface entropy density of $1 / 4 l_{\mathrm{P}}^{2} \sim N / l_{\mathrm{s}}^{2}$.

The fact that the distribution of the soft modes strongly peaks toward the stretched horizon implies the existence of an arbitrariness in splitting them into parts in "high-energy" (i.e., horizon) and low-energy degrees of freedom. In this paper, we adopt a scheme in which the entire BekensteinHawking entropy is associated with the soft modes of the low-energy fields, which was already implied when we took the density of soft-mode states to be $e^{S_{\mathrm{BH}}\left(E_{\mathrm{sott}}\right)}$. This is consistent because we do not describe the internal dynamics among soft modes. In fact, we know that the dynamics of the majority of the soft modes cannot be described by the low-energy theory because they are localized near/at the stretched horizon, where the local intrinsic scale for the dynamics is of order the string scale $1 / l_{\mathrm{s}}$. The internal dynamics of these modes is indeed expected to be nonlocal in the directions along the horizon $[29,30] .{ }^{6}$

Note the crucial role played by the fact that the Bekenstein-Hawking entropy is distributed universally over all the low-energy fields. It is this feature that reconciles the fact that the spacetime picture breaks down at the string scale (at $r \sim r_{\mathrm{s}}$ where $T_{\mathrm{loc}} \sim 1 / l_{\mathrm{s}}$ ) with the fact that $S_{\mathrm{BH}}$ can be written in terms of the Planck length $l_{\mathrm{P}}$ without involving $l_{\mathrm{s}}$.

\section{Bekenstein bound}

An object in field theory in the near horizon region obeys the bound first envisioned by Bekenstein [32] and proved in Ref. [33]

$$
S \lesssim 2 \pi E_{\mathrm{loc}} \rho,
$$

where $S$ and $E_{\text {loc }}$ are the entropy and energy of the object (with $E_{\text {loc }}$ being measured locally at the location of the object), and $\rho$ is the proper distance between the object and

\footnotetext{
${ }^{6}$ This feature may be used to discriminate the hard modes from soft modes near the stretched horizon in a holographic theory at a boundary. A similar separation of modes has been discussed in Ref. [31] for a half-BPS "superstar" geometry in AdS/CFT.
} 
the horizon. We expect that an excitation above the field theory vacuum has

$$
S \gtrsim c,
$$

where $c$ is a number of order a few, ${ }^{7}$ and thus,

$$
c \lesssim S \lesssim 2 \pi E_{\mathrm{loc}} \rho \approx \frac{E_{\mathrm{loc}}}{T_{\mathrm{loc}}}
$$

Here, $T_{\text {loc }}$ is the local Hawking temperature at the location of the object, and we have used the fact that $\rho \approx 1 / 2 \pi T_{\text {loc }}$. We find that an excitation in field theory must have energy

$$
E \gtrsim c T_{\mathrm{H}} \approx O\left(\frac{1}{M l_{\mathrm{P}}^{2}}\right)
$$

as measured in the asymptotic region. This is exactly the condition of being a hard mode.

An alternative way of viewing this is that Eq. (28) says that there are no multiple independent states at the field theory level which correspond to the exponentially many states obtained by exciting soft modes with $E \ll 1 / M l_{\mathrm{P}}^{2}$. This implies that the microstates corresponding to different soft-mode excitations must all be viewed as the same vacuum state at the level of (semiclassical) field theory.

\section{Horizon duality: Chaotic UV dynamics leads to smooth IR spacetime}

The appearance of interior spacetime through Eq. (12) requires a generic black hole state; i.e., the coefficients $c_{n i_{n} a}$ in Eq. (8) take generic values in the $n$ and $i_{n}$ spaces. This implies that the dynamics of the black hole must be chaotic across all low-energy species. Since the intrinsic dynamics of a black hole occurs mostly at the stretched horizon, where the local temperature becomes the string scale, this is translated into the statement about the string dynamics. In particular, the dynamics at the string scale must not have a structure which prevents the universal redistribution of the initial state energy and information over all low-energy species, such as an exact global symmetry. In fact, the breaking of a global symmetry must be strong, parametrically of $O(1)$, at the string scale, in order for the interior spacetime to develop within a reasonable time after the black hole formation (or for the black hole to self-repair sufficiently quickly; see Sec. II D). ${ }^{8}$ This is consistent with earlier observations, e.g., in Refs. [34,35], though it makes a stronger statement about the dynamics at the string scale.

\footnotetext{
${ }^{7}$ Precisely speaking, $S$ in Eq. (28) represents the difference of the von Neumann entropy between the excited and vacuum states. In the analysis below, we coarse-grain the excitation sufficiently so that the resulting mixed state has $S$ sufficiently larger than 1 .

${ }^{8}$ I have recently learned that a similar claim about the strength of global symmetry breaking is being pursued by Cordova, Ohmori, and Rudelius using a different, swampland-related argument.
}

Incidentally, a global symmetry that is nonlinearly realized at the string scale is not constrained by the argument given above. In other words, the required global symmetry breaking at $l_{\mathrm{s}}$ need not be explicit and can be "spontaneous." This suggests that the QCD axion needed to solve the strong $C P$ problem is a string axion (see, e.g., Ref. [36]), since the required quality of the Peccei-Quinn symmetry is very high. If the Peccei-Quinn symmetry were linearly realized at $l_{\mathrm{s}}$, then the above argument would say that it must be explicitly broken with $O(1)$ strength (unless it arises as an approximate accidental symmetry at low energies, resulting from a judicious choice of matter representations under gauge symmetry), which would invalidate the Peccei-Quinn mechanism [37-39].

It is important that the universal dynamics discussed above, leading to generic entanglement between the hard and soft modes, emerges only if the surface of the material composing an object recedes behind the surface at which $T_{\text {loc }} \sim 1 / l_{\mathrm{s}}$, i.e., the stretched horizon. This condition, therefore, can be used as a criterion for differentiating a black hole from normal matter, such as a piece of coal and a regular star. For the latter, the structure of the state does not take the universal form, even though some radiation may be emitted from its surface. The construction of Eq. (12), therefore, does not apply, and hence, no near-empty interior spacetime.

The analysis here reveals an intriguing relation between IR and UV physics: In order to have large-IR — spacetime behind a horizon, its dynamics as viewed from a distancethe UV dynamics-must be chaotic across all low-energy species. For a quasistatic system, this can be stated more quantitatively. Suppose that a spacetime is given with a quasistatic time foliation. For an evolving black hole, this could be done by pulling in "leaves" (equal time hypersurfaces on the boundary) along holographic slices [21]. This pulling-in procedure must halt when gravity/acceleration becomes large, specifically at a surface on which

$$
a \equiv \sqrt{g_{\mu \nu} a^{\mu} a^{\nu}} \sim \frac{1}{l_{\mathrm{s}}},
$$

where

$$
a^{\mu}=n^{\nu} \nabla_{\nu} n^{\mu},
$$

and $n^{\mu}$ is the timelike unit normal to the holographic-or bulk equal time-slice. Because of large acceleration in Eq. (32), physics on this surface is described by the string scale dynamics. It is this dynamics that leads to a wellscrambled state in Eq. (5), or Eq. (8), allowing for the construction of spacetime behind it through Eq. (12).

\section{The paradox of low-energy excitations and its resolution}

In Ref. [14], an important problem was pointed out which was claimed to plague any "state-dependent" construction (see also Ref. [40]). The basic argument is as 
follows: Let us denote the space of pure states with energy $E<E_{0}$ as $\mathcal{H}_{E_{0}}$. For sufficiently large $E_{0}$, we assume that a typical state in $\mathcal{H}_{E_{0}}$ is a black hole with a smooth horizon (which is what the state dependence is supposed to achieve). ${ }^{9}$ Let us now consider unitaries $U_{I}$ of the form $e^{i \sum_{k} \phi_{k}\left(b_{\omega}^{\dagger} b_{\omega}\right)^{k}}$ using appropriately smoothed mode operators whose frequencies (as measured in the asymptotic region) are $\omega \ll \Delta$. These operators, because of the smoothing, change the energy of the state by $\delta E$, where $|\delta E| \ll \Delta$.

One can then show that a typical state in $\mathcal{H}_{E_{0}+\delta E}$ is nearly parallel to a state in $U_{I} \mathcal{H}_{E_{0}}$. Reference [14] argues that this is a contradiction. The claim is that almost all states in $U_{I} \mathcal{H}_{E_{0}}$ must be excited states (stating that otherwise the frozen vacuum argument of Ref. [41] applies), while almost all states in $\mathcal{H}_{E_{0}+\delta E}$ are vacuum states (as those in $\mathcal{H}_{E_{0}}$ ). However, since a state in the latter class is nearly parallel to a state in the former class, this leads to a massive violation of the Born rule.

Our framework addresses this issue in a simple (trivial) manner ${ }^{10}$ : A typical state in $U_{I} \mathcal{H}_{E_{0}}$ is not an excited state, but it is a microstate of the black hole vacuum which is different from those in $\mathcal{H}_{E_{0}}$. Note that given $|\delta E| \ll \Delta$, operating $U_{I}$ on a black hole vacuum state of the form in Eq. (8) corresponds to changing the coefficients $c_{n i_{n} a}$. This simply leads to another black hole vacuum microstate having different coefficients $c_{n i_{n} a}^{\prime}$. In other words, one can repeat the whole construction of Sec. II A with $c_{n i_{n} a}$ replaced with $c_{n i_{n} a}^{\prime}$ to find infalling operators $a_{\xi}^{\prime}, a_{\xi}^{\prime \dagger}$, and $H^{\prime}$, in terms of which the description of a smooth horizon is obtained. Operations involving structures finer than $\Delta$ are not represented by quantum operators in semiclassical theory. (We might say that they correspond to changing the background geometry by minuscule amounts, e.g., $|\delta M| \lesssim \Delta$.) In the context of holography, soft modes are not represented as degrees of freedom specifying states within a code subspace [43] in a way that subsystem recovery is possible.

It is important that exciting a mode with $\omega \gtrsim \Delta$ on a black hole vacuum state does not lead to another black hole vacuum state; it leads to an excited state. For example, one can consider a state in which there are $N$ particles in the zone

\footnotetext{
${ }^{9}$ Originally, this statement was considered in the context of AdS/CFT duality, where the relevant black hole was a large AdS black hole. On the other hand, here we are interested in a black hole in asymptotically flat spacetime (or a small AdS black hole). The analysis below, however, still applies if, instead of CFT Hilbert space, one considers effective Hilbert space $\mathcal{H}_{\Gamma}$ on the boundary $\Gamma$ that is pulled in [21] to become a surface near the black hole, e.g., the $r=c M l_{\mathrm{P}}^{2}$ surface with $c \gtrsim 3$. Possible entanglement between the degrees of freedom inside $\Gamma$ and those outside does not play an important role in the discussion here.

${ }^{10}$ For an attempt to address the issue using causality of AdS spacetime, see Ref. [42].
}

$$
\left|\Psi_{N}\right\rangle \propto \prod_{i=1}^{N}\left(\sum_{\gamma} f_{i, \gamma} b_{\gamma}^{\dagger}\right)|\Psi(M)\rangle,
$$

where $f_{i, \gamma}$ are the weights for producing particle $i$ by superposing creation operators $b_{\gamma}^{\dagger}$ that have frequencies larger than $\Delta$. (Note that for hard particles in the zone, $a_{\gamma}^{\dagger} \approx b_{\gamma}^{\dagger}$.) States like Eq. (34) cannot be written in the form of Eq. (8) with the coefficients $c_{n i_{n} a}$ taking generic values in the $n$ and $i_{n}$ spaces.

In fact, unlike the black hole vacuum states obtained by changing the configuration of soft modes, states obtained by exciting hard modes are not typical in the Hilbert space. Consider the space of all states that are obtained by acting appropriately smoothed hard-mode operators on an element of $\mathcal{H}_{E_{0}}$ and have energies smaller than $E_{0}+\omega$, where $\omega \gtrsim \Delta$. We denote this space by $B_{\omega} \mathcal{H}_{E_{0}}$. One can then show that a typical state $|\psi\rangle$ in $\mathcal{H}_{E_{0}+\omega}$ can be written as

$$
|\psi\rangle=\sin \theta\left|\psi_{\mathrm{exc}}\right\rangle+\cos \theta\left|\psi_{\mathrm{vac}}\right\rangle,
$$

where $\left|\psi_{\text {exc }}\right\rangle$ and $\left|\psi_{\text {vac }}\right\rangle$ are elements of $B_{\omega} \mathcal{H}_{E_{0}}$ and its complement $\mathcal{H}_{E_{0}+\omega} / B_{\omega} \mathcal{H}_{E_{0}}$, respectively, and

$$
\sin ^{2} \theta \sim e^{-\frac{\omega}{T_{\mathrm{H}}}}
$$

We find that a typical state in $\mathcal{H}_{E_{0}+\omega}$ has only negligible overlap with the excited states; i.e., a state obtained by exciting hard modes is atypical in the microscopic Hilbert space. What the semiclassical theory describes is the dynamics of these atypical states.

Another way to phrase the conclusion here is that the Hilbert space $\mathcal{H}_{\text {inf }}$ for the infalling modes, Eqs. (16) and (17), built on each of the black hole and radiation microstates need not overlap with each other. As states excited by $b_{\gamma}^{\dagger}$ 's are atypical and can be discriminated from typical states, states excited by $a_{\xi}^{\dagger}$,s are also atypical in the Hilbert space of the black hole and radiation (of which states with thermal Hawking radiation are typical) and can be discriminated from typical states by energetic consideration. In particular, as long as we are focusing on small excitations, with $\ln \operatorname{dim} \mathcal{H}_{\text {inf }} \sim\left(\mathcal{A}(M) / l_{\mathrm{P}}^{2}\right)^{q}(q<1)$ where $\mathcal{A}(M)$ is the area of the black hole horizon, the existence of $\mathcal{H}_{\text {inf }}$ is entropically negligible, so that it can be attached to each of the black hole vacuum microstates without affecting the entropic consideration of black hole evaporation at the leading order in $l_{\mathrm{P}}^{2} / \mathcal{A}(M)$. In short, our framework does not employ a type of state dependence considered in Ref. [14].

\section{Flow of information and energy: Recapitulation}

The picture emerging from the analyses described above is the following: Gravitational collapse makes a material surface recede until it reaches the point at which the local acceleration of a stationary observer becomes the string 
scale, $a \sim 1 / l_{\mathrm{s}}$. When this happens, chaotic dynamics at the string scale distributes the energy of the material into all low-energy species. Indeed, following the earlier analysis of the entropy, we can integrate the energy density of the soft modes of a single species

$$
\rho_{0}(r) \sim T_{\mathrm{loc}}(r)^{4} \sqrt{1-\frac{2 M l_{\mathrm{P}}^{2}}{r}}
$$

over the zone region $r_{\mathrm{s}} \leq r \leq r_{\mathrm{z}}$ (where the second factor on the right-hand side is the redshift factor), obtaining

$$
E_{\mathrm{soft}, 0} \sim \frac{M l_{\mathrm{P}}^{2}}{l_{\mathrm{s}}^{2}} .
$$

We can therefore reproduce the black hole mass (parametrically) after multiplying the number of low-energy species $N$. Furthermore, due to the energy constraint, the chaotic dynamics generates generic entanglement between the hard and soft modes as in Eqs. (5) and (8). It is this generic entanglement that allows for reconstructing spacetime behind the horizon through the coarse-graining in Eq. (12).

Hawking emission, in the sense of emitting quanta that can be viewed as excitations in semiclassical theory, occurs around the edge of the zone $\left|r^{*}\right| \lesssim O\left(M l_{\mathrm{P}}^{2}\right)$ [13], where

$$
r^{*}=r+2 M l_{\mathrm{P}}^{2} \ln \frac{r-2 M l_{\mathrm{P}}^{2}}{2 M l_{\mathrm{P}}^{2}}
$$

is the tortoise coordinate. An important point is that while the distribution of the soft modes is strongly peaked toward the stretched horizon, there are $O(1)$ degrees of freedomwhich are tiny and fractionally only of $O\left(1 / M^{2} l_{\mathrm{P}}^{2}\right)-$ located around the edge of the zone. The information stored in these degrees of freedom is transferred to far modes (a Hawking quantum) in this region in each timescale of $O\left(M l_{\mathrm{P}}^{2}\right)$. The backreaction creates an ingoing flux of negative energy and negative entropy with respect to the static (Hartle-Hawking [44]) vacuum. Note that the only relevant low-energy fields in this process are those with masses smaller than $T_{\mathrm{H}}$, since $T_{\text {loc }} \sim T_{\mathrm{H}}$ around the edge of the zone. The evaporation of the black hole completes after $O\left(M^{2} l_{\mathrm{P}}^{2}\right)$ steps of this elementary emission process. The entanglement entropy between the black hole and the radiation emitted, $S_{\text {hard+soft }}^{\mathrm{vN}}=S_{\text {rad }}^{\mathrm{vN}}$, follows the Page curve [27], where $S_{A}^{\mathrm{vN}}$ represents the von Neumann entropy of subsystem $A$.

During the evaporation, the structure of entanglement between the hard-mode, soft-mode, and radiation components can be written as [10]

$$
|\Psi(M)\rangle=\sum_{n} \sum_{i_{n}=1}^{\mathcal{N}_{n}} c_{i_{n}}^{n}\left|H_{n}\right\rangle\left|S_{n, i_{n}}\right\rangle\left|R_{n, i_{n}}\right\rangle,
$$

where $\left|H_{n}\right\rangle,\left|S_{n, i_{n}}\right\rangle$, and $\left|R_{n, i_{n}}\right\rangle$ are states of the hard modes, soft modes, and radiation, respectively, and

$$
\mathcal{N}_{n}=\min \left\{e^{S_{\mathrm{BH}}\left(M-E_{n}\right)}, e^{S_{\mathrm{rad}}}\right\},
$$

with $S_{\mathrm{rad}}$ the coarse-grained (thermal) entropy of the radiation. ${ }^{11}$ Here, we have performed the Schmidt decomposition in the space of soft-mode and radiation states for each $n$. This expression makes it clear why the entanglement argument of Ref. [9] does not apply here. The entanglement responsible for unitarity has to do with the summation of the index $i_{n}$ shared between the soft-mode and radiation states (in fact, dominantly the vacuum index $i_{0}$ ), while the entanglement necessary for interior spacetime has to do with the index $n$, and these two can coexist.

The fact that Hawking emission occurs around the edge of the zone has an interesting implication for the nature of the horizon experienced by an infalling observer [10]. Imagine that early Hawking radiation interacts with a detector, leading to different pointer states $\left|d_{I}\right\rangle$. By separating these states from $\left|\phi_{a}\right\rangle$, the state in Eq. (8) can be written as

$$
\begin{aligned}
|\Psi(M)\rangle= & \sum_{n} \sum_{i_{n}=1}^{\mathcal{N}\left(M-E_{n}\right)} \sum_{I} \sum_{a_{I}} c_{n i_{n} I a_{I}}\left|\left\{n_{\alpha}\right\}\right\rangle \\
& \times\left|\psi_{i_{n}}\left(M-E_{n}\right)\right\rangle\left|\phi_{a_{I}}\right\rangle\left|d_{I}\right\rangle .
\end{aligned}
$$

To discuss what the detector finding a particular outcome $I$ will experience later, we focus on the particular branch of the wave function

$$
\begin{aligned}
\left|\Psi_{I}(M)\right\rangle= & \frac{1}{\sqrt{z_{I}}} \sum_{n} \sum_{i_{n}=1}^{\mathcal{N}\left(M-E_{n}\right)} \sum_{a_{I}} c_{n i_{n} I a_{I}}\left|\left\{n_{\alpha}\right\}\right\rangle \\
& \times\left|\psi_{i_{n}}\left(M-E_{n}\right)\right\rangle\left|\phi_{a_{I}}\right\rangle\left|d_{I}\right\rangle,
\end{aligned}
$$

where $z_{I}=\sum_{n} \sum_{i_{n}=1}^{\mathcal{N}\left(M-E_{n}\right)} \sum_{a_{I}}\left|c_{n i_{n} I a_{I}}\right|^{2}$ is the normalization factor. Generically, this does not affect the physics of the black hole, since the structure of Eq. (43) is the same as that of Eq. (8). However, if the detector is carefully set up, it may be fully correlated with a particular configuration $\left\{n_{\alpha}^{\prime}\right\}$ of the hard modes after the measurement, i.e. $c_{n i_{n} I a_{I}} \approx 0$ for $n \neq\left\{n_{\alpha}^{\prime}\right\}$. This seems to mean that when the detector enters the horizon, it would hit a "firewall" because the hard modes lack the necessary entanglement.

However, since the detector can interact with semiclassical Hawking quanta only outside the zone, it can reach the stretched horizon only after time of order $4 M l_{\mathrm{P}}^{2} \ln \left(M l_{\mathrm{P}}\right)$. Therefore, if the equilibrium timescale between the hard and soft modes is of order

$$
t_{\mathrm{eq}}=4 M l_{\mathrm{P}}^{2} \ln \left(M l_{\mathrm{P}}\right)
$$

\footnotetext{
${ }^{11} \mathrm{We}$ assume that the coarse-grained entropies of the three components satisfy $S_{\text {hard }} \ll S_{\text {soft }} \approx S_{\mathrm{BH}}, S_{\text {rad }}$, which is expected to be valid throughout the (essentially) whole history of black hole evolution.
} 
or shorter, then the state of the system (without the detector included) takes the form of Eq. (8) with generic $c_{n i_{n} a}=$ $c_{n i_{n} I a_{I}}$ in the $n$ and $i_{n}$ spaces when the detector enters the stretched horizon. This would imply that an operation acting only on early Hawking radiation-however complicated - cannot destroy the smoothness of the horizon one will see; a black hole self-repairs itself by the time an infaller reaches the horizon.

\section{BEYOND BLACK HOLES}

In this section, we generalize the results of the previous section obtained for an evaporating black hole to other systems. We first discuss how the physics of Rindler spacetime is obtained as a smooth limit of the black hole physics. We then see that essentially all the ideas developed in the previous section can be applied consistently to de Sitter spacetime. We finally consider asymptotically flat spacetime and discuss how some of the ideas developed here may be related to the semiclassical analysis of the asymptotic symmetry structure.

\section{A. Rindler limit}

Rindler spacetime is obtained as a limit of Schwarzschild spacetime

$$
M \rightarrow \infty \quad \text { and } \quad l_{\mathrm{P}}, l_{\mathrm{s}}: \text { fixed }
$$

by focusing on the near horizon region $r \rightarrow 2 M l_{\mathrm{P}}^{2}$, such that the combinations

$$
\rho \equiv 2 \sqrt{2 M l_{\mathrm{P}}^{2}\left(r-2 M l_{\mathrm{P}}^{2}\right)} \quad \text { and } \quad \tau \equiv \frac{R}{4 M l_{\mathrm{P}}^{2}} t
$$

are kept finite, where $R$ is a finite parameter with the dimension of length. The resulting metric is

$$
d s^{2}=-\frac{\rho^{2}}{R^{2}} d \tau^{2}+d \rho^{2}+\sum_{i} d X^{i} d X^{i}
$$

where $X^{i}(i=2,3)$ parametrize the direction parallel to the horizon. Since the Rindler spacetime is obtained by taking the $M l_{\mathrm{P}} \rightarrow \infty$ limit of Schwarzschild spacetime, its entropy is infinite

$$
S_{\text {Rindler }}=\infty,
$$

though the surface entropy density on the stretched horizon is still given by $1 / 4 l_{\mathrm{P}}^{2}$.

There is no direct analog of Hawking emission in the Rindler limit, since the edge of the zone in the original Schwarzschild spacetime is at spatial infinity. ${ }^{12}$ There is, however, an analog of black hole mining $[45,46]$ by which a

\footnotetext{
${ }^{12} \mathrm{We}$ implicitly imagine an IR cutoff $\rho_{\mathrm{IR}} \rightarrow \infty$ so that $\rho_{\mathrm{IR}} / M l_{\mathrm{P}}^{2}<\infty$.
}

physical probe at constant $r$ in the zone observes a thermal bath with temperature $T_{\mathrm{loc}}(r)$, which in the Rindler limit gives

$$
T_{\mathrm{loc}}(\rho)=\lim _{M \rightarrow \infty} \frac{T_{\mathrm{H}}}{\sqrt{1-\frac{2 M l_{\mathrm{P}}^{2}}{r}}}=\frac{1}{2 \pi \rho} .
$$

This is the well-known Unruh effect $[25,47,48]$. While mining allows us to extract information about a black hole vacuum, the Unruh effect is expected not to give any information about a microstate of the Rindler/Minkowski vacuum. This is ensured by Eq. (48); since extracting information about a scrambled system requires accessibility to more than half of its entropy [27,29], no finite size detector can extract such information.

As in the case of a black hole, semiclassical theory in a Rindler wedge describes microscopic dynamics of only the hard modes, whose locally measured energies are sufficiently larger than $T_{\text {loc }}(\rho)$. The other degrees of freedom, the soft modes, are described only statistically. Denoting the states of the hard modes by $\left|\left\{n_{\alpha}\right\}\right\rangle$, microstates of the vacuum can be written in the form of Eq. (5). The construction of mirror operators and "interior spacetime," i.e., the other side of the Rindler horizon, goes as in the black hole case (by first taking $M$ finite and then sending it to infinity). An important difference, however, is that since there is no far mode; the mirror space is constructed purely out of the soft modes.

Another consequence of Eq. (48) is that the scrambling time $[29,30]$ of Rindler spacetime is infinite

$$
\tau_{\text {scr }} \approx O\left(\rho \ln S_{\text {Rindler }}\right) \rightarrow \infty .
$$

This has an operational meaning. It implies that negative energy-entropy excitations generated by backreaction of the Unruh effect and entangled with the detector, do not relax in any finite time. If we reduce the acceleration characterizing the Rindler description, then this entanglementinformation about the other side of the horizon - can be retrieved in the Rindler wedge. While this can be viewed as an analog of information retrieval from a black hole, Eq. (48) implies that the retrieved information is not scrambled. This is consistent with the inertial frame description, which implies that the negative energy-entropy excitations, which can be viewed as particles emitted from the detector in an inertial frame [49], are not thermalized when they reappear from the receding Rindler horizon.

\section{B. de Sitter spacetime}

A consistent microscopic description of de Sitter spacetime is not yet known. There are, however, several proposals aiming toward it. In particular, a description based on a holographic screen seems promising for describing cosmological de Sitter spacetime [21,50], at least when the spacetime deviates-even slightly-from 
the pure de Sitter vacuum (e.g., by the existence of another energy component beyond the cosmological constant). Here instead of committing to a particular proposal, we assume the existence of a consistent description of (approximate) de Sitter spacetime and study what the most straightforward extension of the black hole picture would imply for such a description. For related descriptions of de Sitter spacetime based on the static picture, see Refs. [51,52].

The picture of a black hole in a distant frame is analogous to the static description of de Sitter spacetime (with the radius inside out), whose metric is given by

$$
d s^{2}=-\left(1-H^{2} r^{2}\right) d t^{2}+\frac{1}{1-H^{2} r^{2}} d r^{2}+r^{2} d \Omega^{2},
$$

where $H$ is the Hubble parameter. The entropy and the local temperature are given by [53]

$$
S_{\mathrm{GH}}(H)=\frac{\pi}{H^{2} l_{\mathrm{P}}^{2}}, \quad T_{\mathrm{loc}}(r)=\frac{H}{2 \pi} \frac{1}{\sqrt{1-H^{2} r^{2}}} .
$$

Analogous to the black hole case, we separate modes of low-energy quantum fields into hard $\omega \gtrsim \Delta$ and soft $\omega \lesssim \Delta$ modes, where

$$
\Delta \approx O(H)
$$

is taken to be sufficiently, e.g., $O(10)$, larger than $T_{\mathrm{loc}}(0)=H / 2 \pi$, and the frequency $\omega$ and $\Delta$ are both measured at $r=0$.

The energy of the vacuum obtained by integrating the energy density [see Eqs. (37) and (38)] is

$E \sim N \int_{0}^{r_{\mathrm{s}}} T_{\mathrm{loc}}(r)^{4} \sqrt{1-H^{2} r^{2}} \frac{4 \pi r^{2}}{\sqrt{1-H^{2} r^{2}}} d r \sim \frac{1}{H l_{\mathrm{P}}^{2}}$,

where $r_{\mathrm{s}}$ is the location of the stretched horizon (the string length away from the mathematical horizon $r=1 / H$ )

$$
\frac{1}{H}-r_{\mathrm{s}} \sim H l_{\mathrm{s}}^{2}
$$

Requiring that $\partial S_{\mathrm{GH}}(H) / \partial E=1 / T_{\mathrm{loc}}(0)$, the proportionality factor in Eq. (54) is determined to be unity

$$
E=\frac{1}{H l_{\mathrm{P}}^{2}} .
$$

While the relation of this energy-defined at $r=0$ rather than asymptotic infinity - to more conventionally defined energies is not clear, it can be used to obtain a consistent semiclassical description of the system as we see below.

As in the case of a black hole, we regard the entropy $S_{\mathrm{GH}}(H)$ to represent the density of de Sitter microstates, which correspond to different configurations of the soft modes. A microstate representing the de Sitter vacuum is then given by

$$
|\Psi(H)\rangle=\sum_{n} \sum_{i_{n}=1}^{\mathcal{N}(n)} c_{n i_{n}}\left|\left\{n_{\alpha}\right\}\right\rangle\left|\psi_{i_{n}}(n)\right\rangle,
$$

where $\left|\left\{n_{\alpha}\right\}\right\rangle$ are orthonormal states of the hard modes with $\alpha$ collectively denoting the species, frequency, and angularmomentum quantum numbers of a mode, $E_{n}$ is the energy of the state $\left|\left\{n_{\alpha}\right\}\right\rangle$ as measured at $r=0$, and $\left|\psi_{i_{n}}(n)\right\rangle$ are orthonormal states of soft modes which have energy $E-E_{n}=1 / H l_{\mathrm{P}}^{2}-E_{n}$. Since $E \rightarrow E-E_{n}$ can be interpreted as $H \rightarrow H+E_{n} H^{2} l_{\mathrm{P}}^{2}$,

$$
\mathcal{N}(n)=e^{S_{\mathrm{GH}}\left(H+E_{n} H^{2} l_{\mathrm{P}}^{2}\right)} \approx \exp \left(\frac{\pi}{H^{2} l_{\mathrm{P}}^{2}}-\frac{2 \pi E_{n}}{H}\right) .
$$

Assuming generic coefficients $c_{n i_{n}}$, we can trace out soft modes, which yields

$\operatorname{Tr}_{\text {soft }}|\Psi(H)\rangle\left\langle\Psi(H)\left|=\frac{1}{\sum_{n} e^{-\frac{E_{n}}{T_{\mathrm{GH}}}}} \sum_{n} e^{-\frac{E_{n}}{T_{\mathrm{GH}}}}\right|\left\{n_{\alpha}\right\}\right\rangle\left\langle\left\{n_{\alpha}\right\}\right|$.

This is the thermal density matrix with the temperature

$$
T_{\mathrm{GH}}=T_{\mathrm{loc}}(0)=\frac{H}{2 \pi} .
$$

It is remarkable that the understanding of the entropy and temperature in terms of soft modes is carried over without any modification from a black hole to de Sitter spacetime.

States in which hard modes are excited are obtained by acting corresponding creation operators to a de Sitter vacuum microstate. The annihilation and creation operators for hard modes take the form in Eqs. (10) and (11)

$$
\begin{aligned}
& b_{\gamma}=\sum_{n} \sqrt{n_{\gamma}}\left|\left\{n_{\alpha}-\delta_{\alpha \gamma}\right\}\right\rangle\left\langle\left\{n_{\alpha}\right\}\right|, \\
& b_{\gamma}^{\dagger}=\sum_{n} \sqrt{n_{\gamma}+1}\left|\left\{n_{\alpha}+\delta_{\alpha \gamma}\right\}\right\rangle\left\langle\left\{n_{\alpha}\right\}\right| .
\end{aligned}
$$

It is these excitations of hard modes that we perceive as excitations over the de Sitter vacuum at the semiclassical level. The analysis in Sec. II C for atypicality of excited states goes through in the de Sitter case as well.

\section{Outside the de Sitter horizon}

In a realistic cosmological setup, de Sitter spacetime appears approximately, and it is often the case that, in the standard general relativistic description, the region outside the horizon has much richer structure than the simple, plain de Sitter space. For example, if our Universe began by a bubble nucleation in a parent universe, e.g., as one of infinitely many universes created in eternal inflation, then the Penrose diagram takes the form as in Fig. 1. The spacetime outside the de Sitter horizon, or the holographic screen, has a complicated structure involving many other bubble universes, etc. (Note that because our Universe is 


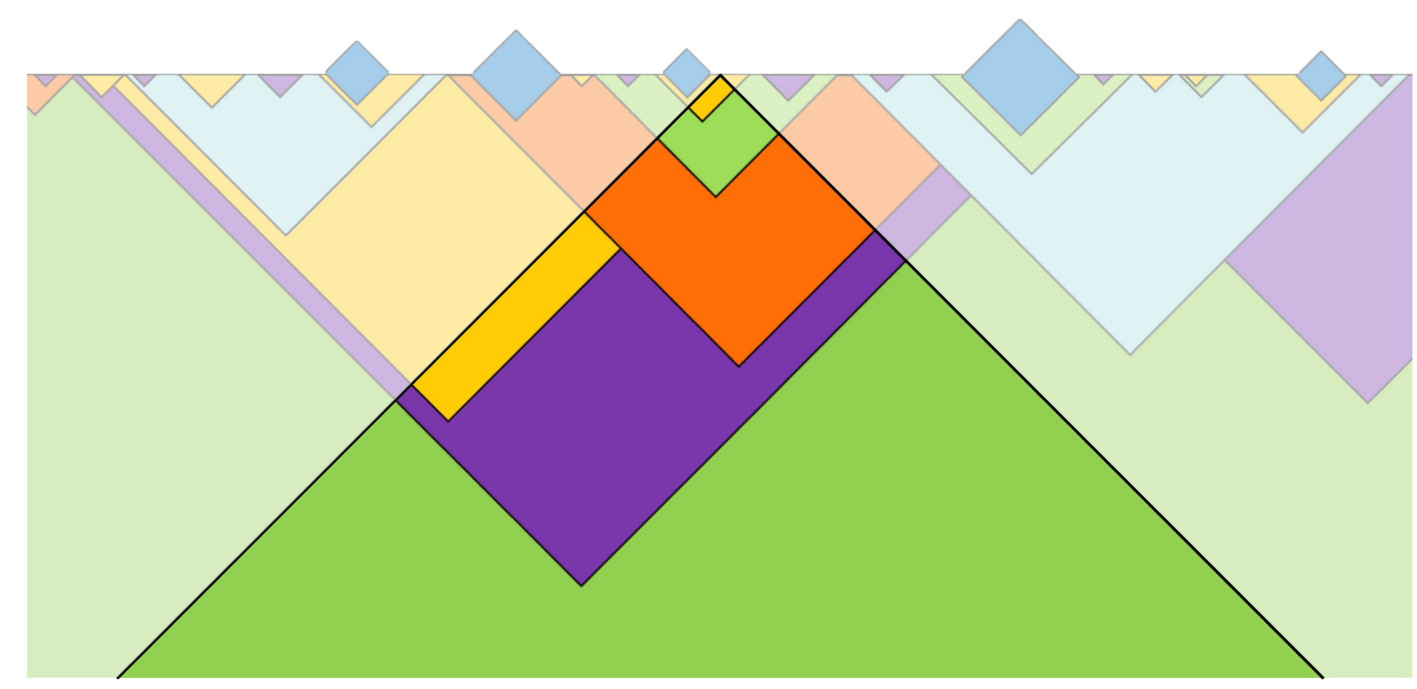

FIG. 1. A schematic depiction illustrating the idea that a single branch of a quantum state describes only the spacetime region accessible by a single observer. The global spacetime of general relativity (the entire region including those represented by lighter colors) arises only as a "pictorial depiction" obtained by patching possible spacetime histories represented by various branches.

not purely de Sitter, e.g., by the existence of a small matter component or an early curvature-dominated phase, the holographic screen at late times lies inside our bubble.) On the other hand, if de Sitter space with a fixed $H$ (specified within the precision allowed by the uncertainty principle) is described as a system with a finite number of degrees of freedom, then how can such a description be consistent with the possible existence of an innumerable variety of complicated spacetimes outside the horizon?

This problem was addressed in Ref. [54], in which it was argued that the general relativistic, global spacetime as in Fig. 1 should (only) be interpreted as a pictorial depiction obtained by "patching" possible different semiclassical spacetime histories one can obtain in a quantum mechanical world, and that each of these histories describes only the spacetime region a single "observer" (timelike curve) can access. This leads to the following picture for the evolution of a quantum state. As a standard scattering experiment converts an initial state with a specific particle configuration into a superposition of terms/branches with different particle configurations, a bubble nucleation-which is a quantum process-makes the state a superposition of branches having different spacetimes, e.g., with different bubbles created at different spacetime locations. Note that the state representing each branch may still have a finite coarse-grained entropy; in particular, for a branch in an approximate de Sitter phase it is given by $S_{\mathrm{GH}}(H)$. This therefore reconciles the finiteness of de Sitter entropy with the "existence" of (infinitely) large spacetime outside the horizon in the general relativistic description.

The framework presented in this paper offers the possibility of making this picture more solid. A specific question addressed is the following: While infinite spacetime outside the (approximate) de Sitter horizon in a single branch may be an illusion, is it not possible to access some part of it, e.g., when the system tunnels into a Minkowski vacuum or if slow-roll inflation ends with its potential energy converted into a different energy component by reheating? How can a framework based on the static picture of Eq. (51) describe such an "information retrieval" process?

Suppose that a state takes the form in Eq. (57) at $t=t_{*}$, possibly with hard modes excited by Eq. (62). As in the case of a black hole, we can erect an effective theory based on the state at $t=t_{*}$ by coarse-graining soft modes

$$
\left.\left.\sum_{i_{n}=1}^{\mathcal{N}(n)} c_{n i_{n}}\left|\psi_{i_{n}}(n)\right\rangle \rightarrow \frac{e^{-\frac{E_{n}}{2 T_{\mathrm{GH}}}}}{\sqrt{\sum_{n} e^{-\frac{E_{n}}{T_{\mathrm{GH}}}}}} \|\left\{n_{\alpha}\right\}\right\rangle\right\rangle
$$

and introducing mirror operators

$$
\begin{aligned}
& \left.\left.\tilde{b}_{\gamma}=\sum_{n} \sqrt{n_{\gamma}} \|\left\{n_{\alpha}-\delta_{\alpha \gamma}\right\}\right\rangle\right\rangle\left\langle\left\{n_{\alpha}\right\} \|,\right. \\
& \left.\tilde{b}_{\gamma}^{\dagger}=\sum_{n} \sqrt{n_{\gamma}+1} \|\left\{n_{\alpha}+\delta_{\alpha \gamma}\right\}\right\rangle\left\langle\left\langle\left\{n_{\alpha}\right\} \| .\right.\right.
\end{aligned}
$$

The vacuum state then becomes

$$
\left.\left.\| \Psi(H)\rangle\rangle=\frac{1}{\sqrt{\sum_{n} e^{-\frac{E_{n}}{T_{\mathrm{GH}}}}}} \sum_{n} e^{-\frac{E_{n}}{2 T_{\mathrm{GH}}}}\left|\left\{n_{\alpha}\right\}\right\rangle \|\left\{n_{\alpha}\right\}\right\rangle\right\rangle .
$$

This allows us to interpret the mirror operators as representing semiclassical modes in the other hemisphere of spherical de Sitter spacetime, with $\tilde{b}_{\gamma}^{\dagger}\left(\tilde{b}_{\gamma}\right)$ creating (annihilating) the mode that is the mirror image of the mode $\gamma$ in the original hemisphere with respect to the bifurcation surface. The correspondence between the black hole and de Sitter cases is as in Table I. Note that for de Sitter 
TABLE I. Correspondence between an evaporating black hole and cosmological de Sitter space.

\begin{tabular}{l}
\hline \hline Microscopic level $\left\{\begin{array}{cc}\text { Evaporating black hole } & \text { Cosmological de Sitter space } \\
\hline \text { Effective theory }\left\{\begin{array}{c}\text { Zone region } \\
\text { Far region }\end{array}\right. & \text { Inside the horizon } \\
\text { Two-sided black hole } \\
\text { The second exterior }\end{array}\right.$ \\
\hline \hline
\end{tabular}

spacetime, there is no region corresponding to the region outside the zone of an evaporating black hole.

As in the black hole case, the effective theory makes manifest the information about semiclassical physics encoded in collective excitations of the soft modes. While the emergent spacetime region outside the horizon is finite, this is sufficient to describe any future development of the branch the system is in. Suppose that at $t=t_{*}$ the coarse-grained state of the system is given by Eq. (66) with excitations on it. This gives the state only on the $t=t_{*}$ hypersurface of the emergent de Sitter space depicted by the solid red line in the Penrose diagram in Fig. 2. Now, the observer associated with the branch (timelike curve at $r=0$ ) can obtain the maximal amount of information about physics occurring outside the de Sitter horizon (the dashed green line) if the system tunnels into a Minkowski vacuum

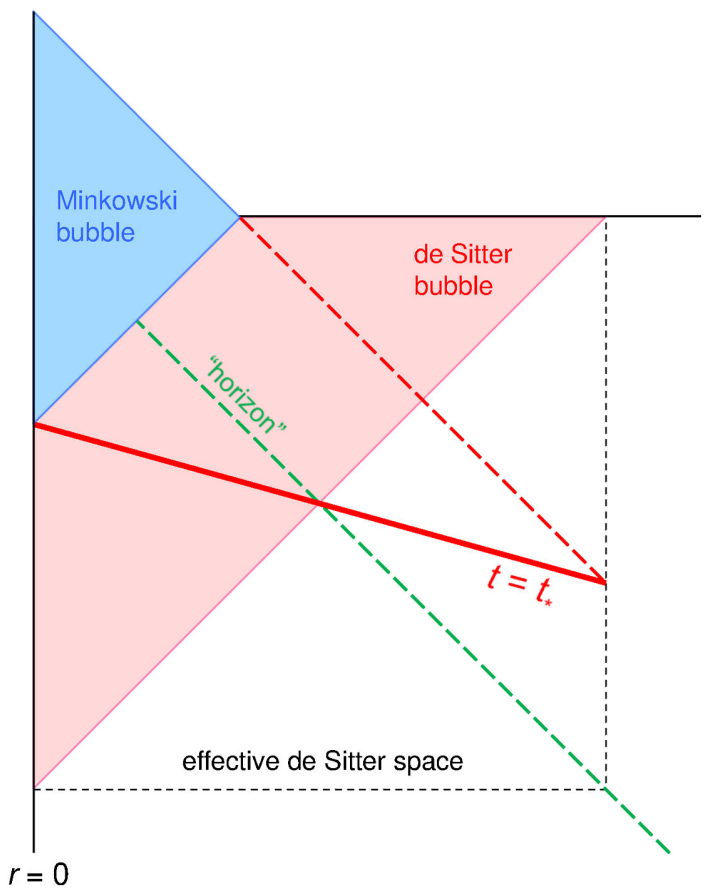

FIG. 2. For a branch having spacetime that appears approximately as a static patch of a de Sitter spacetime, an effective theory can be erected by coarse-graining the soft modes at time $t=t_{*}$. The resulting theory contains a spatial section of an emergent full de Sitter spacetime, including the other side of the horizon. This theory allows for describing any future development of the branch. right after $t=t_{*}$ as depicted in Fig. 2. We find that, even in this case, the knowledge about the state on the $t=t_{*}$ hypersurface is sufficient to fully describe the future signals the observer can receive. In fact, since the argument relies only on causality, this implies that the effective theory can describe the future of the branch (as viewed from the observer) completely, even if its future history is more complicated, e.g., if the system evolves into a superposition of Minkowski bubbles created at different spacetime locations.

As the effective theory of the interior for an evaporating black hole, the effective theory discussed here is not unitary; for example, the future history of a particle that goes outside the causal domain of the $t=t_{*}$ hypersurface cannot be described. As discussed above, however, complete physics the observer at $r=0$ can access is described within the theory. This can be done, for example, by relating the operators in Eqs. (61), (62), (64), and (65) to the annihilation and creation operators for the flat slicing that describes the region inside the de Sitter bubble

$$
\begin{aligned}
& a_{\xi}=\sum_{\gamma}\left(\alpha_{\xi \gamma} b_{\gamma}+\beta_{\xi \gamma} b_{\gamma}^{\dagger}+\zeta_{\xi \gamma} \tilde{b}_{\gamma}+\eta_{\xi \gamma} \tilde{b}_{\gamma}^{\dagger}\right), \\
& a_{\xi}^{\dagger}=\sum_{\gamma}\left(\beta_{\xi \gamma}^{*} b_{\gamma}+\alpha_{\xi \gamma}^{*} b_{\gamma}^{\dagger}+\eta_{\xi \gamma}^{*} \tilde{b}_{\gamma}+\zeta_{\xi \gamma}^{*} \tilde{b}_{\gamma}^{\dagger}\right) .
\end{aligned}
$$

Here, $\xi$ is the label appropriate for the flat slicing, and $\alpha_{\xi \gamma}$, $\beta_{\xi \gamma}, \zeta_{\xi \gamma}$, and $\eta_{\xi \gamma}$ are the Bogoliubov coefficients calculable using the field theory method.

\section{Asymptotically flat spacetime: Relation to BMS}

The analysis of Rindler and de Sitter spacetimes described above has implications for the asymptotic structure of flat spacetime. Let us take the flat space limit of de Sitter spacetime

$$
H \rightarrow 0 \quad \text { and } \quad l_{\mathrm{P}}, l_{\mathrm{s}}: \text { fixed }
$$

with $t$ and $r$ kept finite. In this limit, the horizon is formally sent to infinity, which we may identify as spatial and null infinities of asymptotically flat spacetime. The surface number density of the soft modes there is $1 / 4 l_{\mathrm{P}}^{2}$. In fact, the horizon appears locally as a Rindler horizon.

In the limit of Eq. (69), the soft modes decouple from any experiment performed in a finite spacetime region, as 
indicated by the fact that the local temperature in Eq. (52) goes to zero for finite $r$,

$$
T_{\mathrm{loc}}(r) \rightarrow 0
$$

This implies that the infinite degeneracy of Minkowski vacuum represented by different configurations of the soft modes cannot be observed in any such experiment. Note that the "moduli space" of this degeneracy is huge; it is formally given by the space of a unitary group

$$
\mathcal{M} \approx\left\|U\left(\frac{\mathcal{A}}{4 l_{\mathrm{P}}^{2}}\right)\right\|,
$$

where $\mathcal{A}=4 \pi / H^{2} \rightarrow \infty$ is the area of the IR cutoff surface. Given that each soft-mode degrees of freedom can be arranged to lie at each Planck-sized region on the cutoff surface, we expect that these modes are related to the existence of the BMS group [15-17] and its possible generalization [55] in perturbative quantum gravity. This is consistent with the analysis in Refs. [56,57] and our expectation that a physical process occurring in a finite spacetime region cannot determine the Minkowski vacuum microstate (otherwise, we would have to know a vast amount of information about the initial vacuum microstate to make predictions).

Note that this situation is different from that of a black hole in which the microscopic information stored in the soft modes can be extracted through a Hawking emission or mining process within a finite time. ${ }^{13}$ The difference comes from the fact that a black hole is a finite system interacting with a much larger system. While holography entails that (a vast majority of) the information about a system is stored in its boundary region, the boundary of a black hole is located in the bulk of ambient space reflecting interactions between the two systems. This allows for converting soft, microscopic information of the black hole into different configurations of the hard modes in the ambient space (or in the zone for a mining process), as was discussed in Sec. II.

\section{DISCUSSION: OBSERVABLES IN A QUANTUM WORLD}

We have seen that the formation of a horizon in quantum gravity is accompanied by the emergence of the soft modes arising from the large redshift. While these modes cannot

\footnotetext{
${ }^{13} \mathrm{~A}$ proposal relating black hole information to BMS soft hair has been made in Ref. [58]. Our framework is different from this. The black hole soft modes here contain a structure analogous to but are not associated with the soft BMS charges at infinity. In particular, the BMS symmetry or its extension at infinity does not constrain the product of black hole evaporation. See also Refs. [56,57] for a relevant discussion.
}

be discriminated temporarily by a semiclassical probe in the original reference frame, they play an essential role in describing the "other side" of the horizon, for example, the interior of an evaporating black hole and the outside of a de Sitter horizon. These regions are described by effective theories in which specific operators involving soft modes, e.g., those in Eqs. (14), (15), (64), and (65), play the role of annihilation and creation operators in the effective theories. The construction in Secs. II A and III B guarantees that these operators always exist. One might, however, still ask what selects them as "good operators" to describe the system, in particular, the fate of an object entering the region behind a horizon. This has to do with the issue of the quantum-to-classical transition.

\section{A. Quantum-to-classical transition: Emergence of the Born rule}

Quantum mechanics is formulated to give probabilistic predictions for a measurement of a quantum system performed by a classical observer, whose existence is implicit in the Born rule. Since the division between the quantum system and the surrounding classical world is arbitrary, we expect that the latter arises from more fundamental, intrinsically quantum mechanical substances. While the precise mechanism for how a classical world emerges in quantum mechanics is unknown, it seems reasonable to expect that it has to do with amplification of information [59-61], given that one of the most characteristic features of a classical system is the robustness of information.

A fundamental question about the emergence of a classical world is if it requires an infinitely large environment leading to truly irreversible decoherence, or if a sufficiently large environment holding (enormously) proliferated information is enough. ${ }^{14}$ If the former is true, then we might be able to declare that the only meaningful description of a system is the boundary one. On the other hand, in the latter case, we expect that a description of the black hole interior must make sense, at least for a large enough black hole. In the rest of the paper, we assume (as we have done so far) that the interior of a black hole can be consistently described in quantum mechanics.

In the standard treatment of quantum mechanics, it is customary to postulate that any Hermitian operator acting on the Hilbert space of the observed system is measurable. This is reasonable if the surrounding system making observations has large resources, e.g., enough energy and controllability, so that the outcome of an observation associated with any such operator can be amplified and classicalized. However, if the Hilbert space contains spacetime degrees of freedom, i.e., soft modes, then a process whose effects are fully contained in the corresponding

\footnotetext{
${ }^{14}$ In cosmology, the former situation is realized in the scenarios in Refs. [54,62], while the scenario in Ref. [63] requires the latter.
} 
spacetime region cannot measure everything about them, since there are not enough degrees of freedom within which outcomes are amplified. For example, to measure all Hermitian operators acting on the soft modes of a black hole, we need to couple the black hole to a large external system, e.g., spacetime outside the zone, and use a process, e.g., Hawking emission, that allows for the amplificationand hence, classicalization - of all possible outcomes.

The interior picture does not have such an external system, ${ }^{15}$ and thus, only a small portion of the operators are observable in the sense that they can be used in the Born rule by a classical observer who is a part of the system. Suppose that the vacuum state at the coarse-grained level is given by

$$
\left.\| \Psi\rangle=\frac{1}{\sqrt{\sum_{n} e^{-\frac{E_{n}}{T}}}} \sum_{n} e^{-\frac{E_{n}}{2 T}+i \varphi_{n}}\left|\left\{n_{\alpha}\right\}\right\rangle \|\left\{n_{\alpha}\right\}\right\rangle,
$$

where $\varphi_{n}=\varphi_{\left\{n_{\alpha}\right\}}$ are phases. The construction in Secs. II A and III B suggests that the observables correspond to Hermitian operators constructed out of the original creation and annihilation operators $b_{\gamma}$ and $b_{\gamma}^{\dagger}$ as well as the mirror operators

$$
\begin{aligned}
& \left.\left.\tilde{b}_{\gamma}=\sum_{n} \sqrt{n_{\gamma}} e^{i\left(\varphi_{n_{-}}^{\prime}-\varphi_{n}^{\prime}\right)} \|\left\{n_{\alpha}-\delta_{\alpha \gamma}\right\}\right\rangle\right\rangle\left\langle\left\{n_{\alpha}\right\} \|,\right. \\
& \left.\left.\tilde{b}_{\gamma}^{\dagger}=\sum_{n} \sqrt{n_{\gamma}+1} e^{i\left(\varphi_{n_{+}}^{\prime}-\varphi_{n}^{\prime}\right)} \|\left\{n_{\alpha}+\delta_{\alpha \gamma}\right\}\right\rangle\right\rangle\left\langle\left\{n_{\alpha}\right\} \|,\right.
\end{aligned}
$$

where $n_{ \pm} \equiv\left\{n_{\alpha} \pm \delta_{\alpha \gamma}\right\}$ and

$$
\varphi_{n}^{\prime}=\varphi_{n} \quad \forall n
$$

(because the phases $\varphi_{n}$ can be absorbed by the redefinition of the coarse-grained states $\left.\left.\left.\left.\left.\|\left\{n_{\alpha}\right\}\right\rangle\right\rangle \rightarrow e^{-i \varphi_{n}} \|\left\{n_{\alpha}\right\}\right\rangle\right\rangle\right)$. In fact, we can construct appropriately localized field operators out of these operators, and the generator of time evolution relating them can be given by Eq. (18). The state in Eq. (72) is then the ground state of this generator.

The fact that the operators in Eqs. (73) and (74) provide the right building blocks is intuitive to understand. As viewed from these operators-more precisely, operators related to them by a Bogoliubov transformation-the state in Eq. (72) represents a smooth spacetime, and we empirically know that information about the outcome of a measurement performed in the vicinity of such a state is appropriately classicalized when evolved by the generator of which Eq. (72) is the ground state. On the other hand, if we choose the "annihilation and creation operators" of the form of Eqs. (73) and (74) but violating Eq. (75) in a generic manner, then the state in Eq. (72) appears as a "firewall state" with cutoff scale excitations, and Hermitian operators constructed out of them would not represent observables that can be used by a classical observer in the Born rule.

Given the role locality plays in the process of information amplification [59-61], it seems reasonable to conjecture that the emergence of a classical world -in particular, a classical observer who can use the Born rule to predict the outcome of a measurement-requires that there exists a Hilbert space basis in which the Hamiltonian takes a local (nearest neighbor) form and the relevant states are sufficiently near the ground state so that the smooth spacetime picture is available. This implies, for example, that for a state of the form in Eq. (8), the annihilation and creation operators appearing in observables must be taken to be

$$
\begin{aligned}
\tilde{b}_{\gamma}= & \left(\sum_{n^{\prime}} e^{-\frac{E_{n^{\prime}}}{T_{\mathrm{H}}}}\right) \sum_{n} \sqrt{n_{\gamma}} e^{-\frac{E_{n_{-}+E_{n}}}{2 T_{\mathrm{H}}}} \\
& \times \sum_{i_{n_{-}}=1}^{\mathcal{N}\left(M-E_{n_{-}}\right)} \sum_{i_{n}=1}^{\mathcal{N}\left(M-E_{n}\right)} \sum_{a} \sum_{b} c_{n_{-} i_{n_{-}} a} c_{n i_{n} b}^{*}\left|\psi_{i_{n_{-}}}\left(M-E_{n_{-}}\right)\right\rangle\left|\phi_{a}\right\rangle\left\langle\psi_{i_{n}}\left(M-E_{n}\right)\right|\left\langle\phi_{b}\right|, \\
\tilde{b}_{\gamma}^{\dagger}= & \left(\sum_{n^{\prime}} e^{-\frac{E_{n^{\prime}}}{T_{\mathrm{H}}}}\right) \sum_{n} \sqrt{n_{\gamma}+1} e^{-\frac{E_{n_{+}+E_{n}}}{2 T_{\mathrm{H}}}} \\
& \times \sum_{i_{n_{+}}=1} \sum_{i_{n}=1}^{\mathcal{N}\left(M-E_{n_{+}}\right)} \sum_{a} \sum_{b} c_{n_{+} i_{n_{+}} a} c_{n i_{n} b}^{*}\left|\psi_{i_{n_{+}}}\left(M-E_{n_{+}}\right)\right\rangle\left|\phi_{a}\right\rangle\left\langle\psi_{i_{n}}\left(M-E_{n}\right)\right|\left\langle\phi_{b}\right|,
\end{aligned}
$$

\footnotetext{
${ }^{15}$ The picture involves early Hawking radiation in the ambient space, but this does not provide independent degrees of freedom that can be used for amplification, as can be seen from the entanglement structure in Eq. (40).
}

or those related in a simple way with these operators (e.g., by a Bogoliubov transformation or time evolution). While we have not proven it, the conjecture seems plausible and would explain why the construction in Secs. II A and III B 
is adopted when describing the physics perceived by an object crossing the horizon. It is the construction that makes manifest the observables to which the object can apply the Born rule.

The issue discussed here of selecting appropriate observables is irrelevant in asymptotically flat or AdS spacetimes because of the existence of an infinite amount of degrees of freedom at asymptotic infinity, to which the information can be amplified. (In fact, one can view an asymptotically flat spacetime as a proxy of a sufficiently isolated system, with a physical observer "modeled" by the soft modes at infinity.) In particular, the standard $S$ matrix paradigm does not-because it need not-address the issue. However, cosmological spacetimes generally do not have such "infinitely powerful observers," reflecting the fact that the system is effectively finite. Therefore, unless we resort to some infinite degrees of freedom somewhere, discussion about the quantum-toclassical transition-and hence, the emergence of the
Born rule - cannot be avoided. This indeed seems to be one of the most fundamental problems in understanding the world we live in.

\section{ACKNOWLEDGMENTS}

I am grateful to Pratik Rath and Arvin ShahbaziMoghaddam for useful conversations. I also thank the Yukawa Institute for Theoretical Physics at Kyoto University for holding the workshop YITP-T-19-03 "Quantum Information and String Theory 2019," during which a part of this work was carried out. This work was supported in part by the Department of Energy, Office of Science, Office of High Energy Physics under Contract No. DE-AC02-05CH11231 and Award No. DESC0019380, by the National Science Foundation under Grant No. PHY-1521446, by MEXT KAKENHI Grant No. $15 \mathrm{H} 05895$, and by World Premier International Research Center Initiative (WPI Initiative), MEXT, Japan.
[1] J. D. Bekenstein, Black holes and entropy, Phys. Rev. D 7, 2333 (1973).

[2] S. W. Hawking, Particle creation by black holes, Commun. Math. Phys. 43, 199 (1975); Erratum, Commun. Math. Phys. 46, 206 (1976).

[3] G. 't Hooft, in Salamfestschrift, edited by A. Ali, J. Ellis, and S. Randjbar-Daemi (World Scientific, Singapore, 1994), p. 284.

[4] L. Susskind, The world as a hologram, J. Math. Phys. (N.Y.) 36, 6377 (1995).

[5] R. Bousso, A covariant entropy conjecture, J. High Energy Phys. 07 (1999) 004.

[6] J. Maldacena, The large $N$ limit of superconformal field theories and supergravity, Int. J. Theor. Phys. 38, 1113 (1999); Adv. Theor. Math. Phys. 2, 231 (1998).

[7] S. W. Hawking, Breakdown of predictability in gravitational collapse, Phys. Rev. D 14, 2460 (1976).

[8] S.D. Mathur, The information paradox: A pedagogical introduction, Classical Quantum Gravity 26, 224001 (2009).

[9] A. Almheiri, D. Marolf, J. Polchinski, and J. Sully, Black holes: Complementarity or firewalls?, J. High Energy Phys. 02 (2013) 062.

[10] Y. Nomura, Reanalyzing an evaporating black hole, Phys. Rev. D 99, 086004 (2019).

[11] K. Papadodimas and S. Raju, An infalling observer in AdS/CFT, J. High Energy Phys. 10 (2013) 212.

[12] J. Maldacena and L. Susskind, Cool horizons for entangled black holes, Fortschr. Phys. 61, 781 (2013).

[13] Y. Nomura, F. Sanches, and S. J. Weinberg, Black Hole Interior in Quantum Gravity, Phys. Rev. Lett. 114, 201301 (2015).

[14] D. Marolf and J. Polchinski, Violations of the Born rule in cool state-dependent horizons, J. High Energy Phys. 01 (2016) 008.
[15] H. Bondi, M. G. J. van der Burg, and A. W. K. Metzner, Gravitational waves in general relativity VII. Waves from axisymmetric isolated systems, Proc. R. Soc. A 269, 21 (1962).

[16] R. K. Sachs, Gravitational waves in general relativity VIII. Waves in asymptotically flat space-times, Proc. R. Soc. A 270, 103 (1962).

[17] A. Strominger, On BMS invariance of gravitational scattering, J. High Energy Phys. 07 (2014) 152.

[18] G. Penington, Entanglement wedge reconstruction and the information paradox, arXiv:1905.08255.

[19] A. Almheiri, N. Engelhardt, D. Marolf, and H. Maxfield, The entropy of bulk quantum fields and the entanglement wedge of an evaporating black hole, J. High Energy Phys. 12 (2019) 063.

[20] Y. Nomura, The interior of a unitarily evaporating black hole, arXiv:1911.13120.

[21] Y. Nomura, P. Rath, and N. Salzetta, Pulling the boundary into the bulk, Phys. Rev. D 98, 026010 (2018).

[22] L. Susskind, L. Thorlacius, and J. Uglum, The stretched horizon and black hole complementarity, Phys. Rev. D 48, 3743 (1993).

[23] R. Bousso, Holography in general space-times, J. High Energy Phys. 06 (1999) 028.

[24] Y. Nomura, N. Salzetta, F. Sanches, and S. J. Weinberg, Toward a holographic theory for general spacetimes, Phys. Rev. D 95, 086002 (2017).

[25] W. G. Unruh, Notes on black hole evaporation, Phys. Rev. D 14, 870 (1976).

[26] W. Israel, Thermo field dynamics of black holes, Phys. Lett. 57A, 107 (1976).

[27] D. N. Page, Information in Black Hole Radiation, Phys. Rev. Lett. 71, 3743 (1993). 
[28] G. Dvali, Black holes and large $N$ species solution to the hierarchy problem, Fortschr. Phys. 58, 528 (2010).

[29] P. Hayden and J. Preskill, Black holes as mirrors: Quantum information in random subsystems, J. High Energy Phys. 09 (2007) 120.

[30] Y. Sekino and L. Susskind, Fast scramblers, J. High Energy Phys. 10 (2008) 065.

[31] V. Balasubramanian, D. Berenstein, A. Lewkowycz, A. Miller, O. Parrikar, and C. Rabideau, Emergent classical spacetime from microstates of an incipient black hole, J. High Energy Phys. 01 (2019) 197.

[32] J. D. Bekenstein, Universal upper bound on the entropy to energy ratio for bounded systems, Phys. Rev. D 23, 287 (1981).

[33] H. Casini, Relative entropy and the Bekenstein bound, Classical Quantum Gravity 25, 205021 (2008).

[34] J. Maldacena, S. H. Shenker, and D. Stanford, A bound on chaos, J. High Energy Phys. 08 (2016) 106.

[35] D. Harlow and H. Ooguri, Symmetries in quantum field theory and quantum gravity, arXiv:1810.05338.

[36] P. Svrček and E. Witten, Axions in string theory, J. High Energy Phys. 06 (2006) 051.

[37] M. Kamionkowski and J. March-Russell, Planck scale physics and the Peccei-Quinn mechanism, Phys. Lett. B 282, 137 (1992).

[38] S. M. Barr and D. Seckel, Planck scale corrections to axion models, Phys. Rev. D 46, 539 (1992).

[39] R. Holman, S. D. H. Hsu, T. W. Kephart, E. W. Kolb, R. Watkins, and L. M. Widrow, Solutions to the strong $C P$ problem in a world with gravity, Phys. Lett. B 282, 132 (1992).

[40] D. Harlow, Aspects of the Papadodimas-Raju proposal for the black hole interior, J. High Energy Phys. 11 (2014) 055.

[41] R. Bousso, Violations of the Equivalence Principle by a Nonlocally Reconstructed Vacuum at the Black Hole Horizon, Phys. Rev. Lett. 112, 041102 (2014).

[42] S. Raju, Smooth causal patches for AdS black holes, Phys. Rev. D 95, 126002 (2017).

[43] A. Almheiri, X. Dong, and D. Harlow, Bulk locality and quantum error correction in AdS/CFT, J. High Energy Phys. 04 (2015) 163.

[44] J. B. Hartle and S. W. Hawking, Path integral derivation of black hole radiance, Phys. Rev. D 13, 2188 (1976).

[45] W. G. Unruh and R. M. Wald, Acceleration radiation and generalized second law of thermodynamics, Phys. Rev. D 25, 942 (1982).
[46] A. R. Brown, Tensile Strength and the Mining of Black Holes, Phys. Rev. Lett. 111, 211301 (2013).

[47] S. A. Fulling, Nonuniqueness of canonical field quantization in Riemannian space-time, Phys. Rev. D 7, 2850 (1973).

[48] P. C. W. Davies, Scalar particle production in Schwarzschild and Rindler metrics, J. Phys. A 8, 609 (1975).

[49] W. G. Unruh and R. M. Wald, What happens when an accelerating observer detects a Rindler particle, Phys. Rev. D 29, 1047 (1984).

[50] Y. Nomura, P. Rath, and N. Salzetta, Spacetime from unentanglement, Phys. Rev. D 97, 106010 (2018).

[51] T. Banks, Cosmological breaking of supersymmetry?, Int. J. Mod. Phys. A 16, 910 (2001).

[52] T. Banks, TASI lectures on holographic space-time, SUSY and gravitational effective field theory, arXiv:1007.4001.

[53] G. W. Gibbons and S. W. Hawking, Cosmological event horizons, thermodynamics, and particle creation, Phys. Rev. D 15, 2738 (1977).

[54] Y. Nomura, Physical theories, eternal inflation, and the quantum universe, J. High Energy Phys. 11 (2011) 063.

[55] M. Campiglia and A. Laddha, Asymptotic symmetries and subleading soft graviton theorem, Phys. Rev. D 90, 124028 (2014).

[56] M. Mirbabayi and M. Porrati, Dressed Hard States and Black Hole Soft Hair, Phys. Rev. Lett. 117, 211301 (2016).

[57] R. Bousso and M. Porrati, Soft hair as a soft wig, Classical Quantum Gravity 34, 204001 (2017).

[58] S. W. Hawking, M. J. Perry, and A. Strominger, Soft Hair on Black Holes, Phys. Rev. Lett. 116, 231301 (2016).

[59] H. Ollivier, D. Poulin, and W. H. Zurek, Objective Properties from Subjective Quantum States: Environment as a Witness, Phys. Rev. Lett. 93, 220401 (2004).

[60] R. Blume-Kohout and W. H. Zurek, Quantum Darwinism: Entanglement, branches, and the emergent classicality of redundantly stored quantum information, Phys. Rev. A 73, 062310 (2006).

[61] Y. Nomura, Quantum mechanics, spacetime locality, and gravity, Found. Phys. 43, 978 (2013).

[62] R. Bousso and L. Susskind, Multiverse interpretation of quantum mechanics, Phys. Rev. D 85, 045007 (2012).

[63] Y. Nomura, Static quantum multiverse, Phys. Rev. D 86, 083505 (2012). 